NASA/CR-1999-209361

ICASE Report No. 99-27

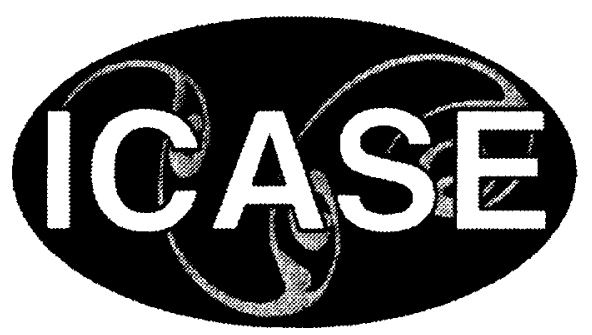

\title{
A High Order Discontinuous Galerkin Method for 2D Incompressible Flows
}

Jian-Guo Liu

University of Maryland, College Park, Maryland

Chi-Wang Shu

Brown University, Providence, Rhode Island

Institute for Computer Applications in Science and Engineering

NASA Langley Research Center

Hampton, VA

Operated by Universities Space Research Association

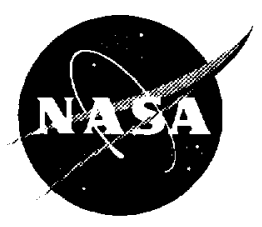

National Aeronautics and

Space Administration

Langley Research Center

Hampton, Virginia 23681-2199 
Available from the following:

NASA Center for AeroSpace Information (CASI)

7121 Standard Drive

Hanover, MD 21076-1320

(301) 621-0390
National Technical Information Service (NTIS)

5285 Port Royal Road

Springfield, VA 22161-2171

(703) 487-4650 


\title{
A HIGH ORDER DISCONTINUOUS GALERKIN METHOD FOR 2D INCOMPRESSIBLE FLOWS
}

\author{
JIAN-GUO LIU* ANI) CHI-WANG SHU⿱一土
}

\begin{abstract}
In this paper we introduce a high order discontinuous Galerkin method for two dimensional incompressible flow in vorticity streamfunction formulation. The momentum equation is treated explicitly, utilizing the efficiency of the discontinuous Galerkin method. The streamfunction is obtained by a standard Poisson solver using continuous finite elements. There is a natural matching between these two finite element spaces, since the normal component of the velocity field is continuous across element boundaries. This allows for a correct upwinding gluing in the discontinuous Galerkin framework, while still maintaining total energy conservation with no numerical dissipation and total enstrophy stability. The method is suitable for inviscid or high Reynolds number flows. Optimal error estimates are proven and verified by numerical experiments.
\end{abstract}

Key words. incompressible flow, discontinuous Galerkin, high order accuracy

Subject classification. Applied and Numerical Mathematics

1. Introduction and the Setup of the Scheme. We are interested in solving the following $2 \mathrm{D}$ time dependent incompressible Euler equations in vorticity streamfunction formulation:

$$
\begin{aligned}
& \omega_{t}+\nabla \cdot(\mathbf{u} \omega)=0 \\
& \Delta \psi=\omega, \quad \mathbf{u}=\nabla^{\perp} \psi, \\
& \mathbf{u} \cdot \mathbf{n}=\text { given on } \partial \Omega,
\end{aligned}
$$

where $\nabla^{\perp}=\left(-\partial_{y}, \partial_{x}\right)$. Notice that the boundary condition, plus the fact that $\mathbf{u} \cdot \mathbf{n}=\frac{\partial \psi}{\partial \tau}$, recovers $\psi$ on the boundary (up to a constant) in a simple connected domain

$$
\left.\psi\right|_{a \Omega}=\psi_{b}
$$

We are also interested in solving the Navier-Stokes equations with high Reynolds numbers Re $\gg 1$ :

$$
\begin{aligned}
& \omega_{t}+\nabla \cdot(\mathbf{u} \omega)=\frac{1}{\operatorname{Re}} \Delta \omega \\
& \Delta \psi=\omega, \quad \mathbf{u}=\nabla^{\perp} \psi, \\
& \mathbf{u}=\text { given on } \partial \Omega .
\end{aligned}
$$

The boundary condition is now (1.2) plus the non-slip type boundary condition:

$$
\left.\frac{\partial \psi}{\partial \mathbf{n}}\right|_{\partial \Omega}=\mathbf{u}_{b, \tau} .
$$

For simplicity, we only consider the no-flow, no-slip boundary conditions $\psi_{b}=0, \mathbf{u}_{b, \tau}=0$ and periodic boundary conditions.

\footnotetext{
* Department of Mathematics, University of Maryland, College Park, Maryland 20742. E-mail: jliu@math.umd.edu. Research supported by NSF grant DMS-9805621.

$\dagger$ Division of Applied Mathematics, Brown University, Providence, Rhode Island 02912. E-mail: shu@cfm.brown.edu. Research supported by ARO grant DAAG55-97-1-0318, NSF grants DMS-9804985, ECS-9627849 and INT-9601084, NASA Langley grant NAG-1-2070 and Contract NAS1-97046 while this author was in residence at ICASE, NASA Langley Research Center, Hampton, VA 23681-2199, and AFOSR grant F49620-99-1-0077.
} 
We first emphasize that, for Euler equations (1.1) and high Reynolds number (Re $\gg 1$ ) Navier-Stokes equations (1.3), it is advantageous to treat both the convective terms and the viscous terms explicitly. The methods discussed in this paper are stable under standard CFL conditions. Since the momentum equation (the first equation in (1.1) and (1.3)) is treated explicitly in the discontinuous Galerkin framework, there is no global mass matrix to invert, unlike conventional finite element methods. This makes the method highly efficient for parallel implementation, see for example [2]. As any finite element method, our approach has the flexibility for complicated geometry and boundary conditions. The method is adapted from the Runge-Kutta discontinuous Galerkin methods discussed by Cockburn et al. in a series of papers [7], [8], [9], [10], [11], [12], $[20]$ and $[6]$.

The main difficulties in solving incompressible flows are the incompressibility condition and boundary conditions. The incompressibility condition is global and is thus solved by the standard Poisson solver for the streamfunction $\psi$ using continuous finite elements. One advantage of our approach is that there is no matching conditions needed for the two finite element spaces for the vorticity $\omega$ and for the streamfunction v. The incompressibility condition, represented by the streamfunction $\psi$, is exactly satisfied pointwise, and is naturally matched with the convective terms in the momentum equation. The normal velocity $\mathbf{u} \cdot \mathbf{n}$ is automatically continuous along any element boundary, allowing for correct upwinding for the convective terms and still maintaining a total energy conservation and total enstrophy stability.

There is an easy proof for stability, both in the total enstrophy and in the total energy, which does not depend on the regularity of the exact solutions. For smooth solutions error estimates can be obtained.

Our method, as it stands, can only compute 2D flows. Similar approach for the primitive variable formulation, suitable for $3 \mathrm{D}$ calculations, is under investigation.

We do not advocate our method for modest or low Reynolds number flows. In such regime viscosity terms should be treated implicitly for efficiency. This is a much more challenging task in terms of space matching characterized by the Babuška-Brezzi-Ladyzenskaja condition, projection type methods, and global vorticity boundary conditions, see for example [3], [17], [25], [27], [18], [19], [24], etc.

For convection dominated flows, as we are interested in this paper, we mention the work of Bell et al. [1] for second order Godunov type upwinding methods, see also Levy and Tadmor [22] and E and Shu [16]. This is still an active field for research.

We now describe the setup of the scheme. We start with a triangulation $\mathcal{T}_{h}$ of the domain $\Omega$, consisting of polygons of maximum size (diameter) $h$, and the following two approximation spaces

$$
V_{h}^{k}=\left\{v:\left.v\right|_{K} \in P^{k}(K), \forall K \in \mathcal{T}_{h}\right\}, \quad W_{0, h}^{k}=V_{h}^{k} \cap C_{0}(\Omega),
$$

where $P^{k}(K)$ is the set of all polynomials of degree at most $k$ on the cell $K$.

For the Euler equations (1.1), the numerical method is defined as follows: find $\omega_{h} \in V_{h}^{k}$ and $\psi_{h} \in W_{0, h}^{k}$, such that

$$
\begin{gathered}
\left\langle\partial_{t} \omega_{h} v\right\rangle_{K}-\left\langle\omega_{h} \mathbf{u}_{h} \cdot \nabla v\right\rangle_{K}+\sum_{e \in \partial K^{k}}\left\langle\mathbf{u}_{h} \cdot \mathbf{n} \widehat{\omega_{h}} v^{-}\right\rangle_{e}=0, \quad \forall v \in V_{h}^{k}, \\
-\left\langle\nabla \psi_{h} \cdot \nabla \varphi\right\rangle=\left\langle\omega_{h} \varphi\right\rangle, \quad \forall \varphi \in W_{0, h}^{k},
\end{gathered}
$$

with the velocity field obtained from the stream function by

$$
\mathbf{u}_{h}=\nabla^{\perp} \psi_{h} .
$$


Here $\langle\cdot\rangle$ is the usual integration over either the whole domain $\Omega$ or a subdomain denoted by a subscript. Same thing for $\|\cdot\|$ for the $L^{2}$ norm.

Notice that the normal velocity $\mathbf{u}_{h} \cdot \mathbf{n}$ is continuous across any element boundary $e$, but both the solution $\omega_{h}$ and the test function $v$ are discontinuous there. We take the values of the test function from within the element $K$, denoted by $v^{-}$. The solution at the edge is taken as a single valued flux $\widehat{w_{h}}$, which can be either a central or a upwind biased average. For example, the central flux is defined by

$$
\widehat{\omega_{h}}=\frac{1}{2}\left(\omega_{h}^{+}+\omega_{h}^{-}\right)
$$

where $\omega_{h}^{+}$is the value of $\omega_{h}$ on the edge e from outside $K$, the complete upwind flux is defined by

$$
\widehat{\omega_{h}}= \begin{cases}\omega_{h}^{-} & \text {if } \mathbf{u}_{h} \cdot \mathbf{n} \geq 0, \\ \omega_{h}^{+} & \text {if } \mathbf{u}_{h} \cdot \mathbf{n}<0 .\end{cases}
$$

and the Lax-Friedrichs upwind biased flux is defined by

$$
\mathbf{u}_{h} \cdot \mathbf{n} \widehat{\omega_{h}}=\frac{1}{2}\left[\mathbf{u}_{h} \cdot \mathbf{n}\left(\omega_{h}^{+}+\omega_{h}^{-}\right)-\alpha\left(\omega_{h}^{+}-\omega_{h}^{-}\right)\right]
$$

where $\alpha$ is the maximum of $\left|\mathbf{u}_{h} \cdot \mathbf{n}\right|$ either locally (local Lax-Friedrichs) or globally (global Lax-Friedrichs).

We remark that, for general boundary conditions (1.2), the space $W_{0, h}^{k}$ in (1.5) should be modified to take the boundary value into consideration. Moreover, additional physical vorticity boundary condition for any inlet should be known.

Navier-Stokes equations (1.3) can be handled in a similar way, with the additional viscous terms treated by the local discontinuous Galerkin technique in [12], and with a local vorticity boundary condition in [13]. The detail is left to Sect. 3. Sect. 2 is devoted to the discussion of stability and error estimates for the Euler equations. Accuracy check and numerical examples are given in Sect. 4. Concluding remarks are given in Sect. 5 .

2. Stability and Error Estimates for the Euler Equations. For stability analysis, we take the test function $v=\omega_{h}$ in (1.6), obtaining

$$
\frac{d}{d t} \frac{1}{2}\left\|\omega_{h}\right\|_{K}^{2}-\frac{1}{2}\left\langle\nabla \cdot\left(\omega_{h}^{2} \mathbf{u}_{h}\right)\right\rangle_{K}+\sum_{e \in \partial K^{\prime}}\left\langle\mathbf{u}_{h} \cdot \mathbf{n} \widehat{\omega_{h}} \omega_{h}^{-}\right\rangle_{e}=0
$$

where we have used the exact incompressibility condition satisfied by $\mathbf{u}_{h}$ for the second term. Performing an integration by parts for the second term, we obtain

$$
\frac{d}{d t} \frac{1}{2}\left\|\omega_{h}\right\|_{K^{\prime}}^{2}+\sum_{e \in \partial K^{\prime}}\left\langle\mathbf{u}_{h} \cdot \mathbf{n}\left(\widehat{\omega_{h}} \omega_{h}^{-}-\frac{1}{2}\left(\omega_{h}^{-}\right)^{2}\right)\right\rangle_{e}=0 .
$$

Now, using the fact that

$$
\omega^{-}=\bar{\omega}-\frac{1}{2}[\omega], \quad\left(\omega^{-}\right)^{2}=\overline{\omega^{2}}-\bar{\omega}[\omega]
$$

where

$$
\bar{\omega}=\frac{1}{2}\left(\omega^{+}+\omega^{-}\right), \quad[\omega]=\omega^{+}-\omega^{-},
$$

we obtain

$$
\frac{d}{d t} \frac{1}{2}\left\|\omega_{h}\right\|_{K}^{2}+\sum_{e \in \partial K}\left\langle\mathbf{u}_{h} \cdot \mathbf{n}\left(\widehat{\omega_{h}} \overline{\omega_{h}}-\frac{1}{2} \overline{\omega_{h}^{2}}\right)\right\rangle_{e}+\frac{1}{2} \sum_{e \in \partial K}\left\langle\mathbf{u}_{h} \cdot \mathbf{n}\left[\omega_{h}\right]\left(\overline{\omega_{h}}-\widehat{\omega_{h}}\right)\right\rangle_{e}=0
$$


Notice that the second term is of opposite sign for adjacent elements sharing a common edge $e$, hence it becomes zero after summing over all the elements $K$ (using the no-flow boundary condition on the physical boundary). The third term is the numerical dissipation: when $\widehat{\omega_{h}}$ is taken as the central flux (1.9), the third term is exactly zero; for the upwind flux (1.10), the third term becomes a positive quantity

$$
\frac{1}{4} \sum_{t \in \partial h^{\prime}}\left\langle\left|\mathbf{u}_{h} \cdot \mathbf{n}\right|\left[\omega_{h}\right]^{2}\right\rangle_{e}
$$

which is the total enstrophy dissipation. The effect of this is to control the size of the jump across the element interface and essentially "gluing" the solution there. Other upwind biased fluxes such as the Lax-Friedrichs flux (1.11) would produce a similar positive term as the total enstrophy dissipation. For smooth flows these jumps are of the order $O\left(h^{k}\right)$ within the truncation error of the scheme. We thus obtain the the following enstrophy inequality

$$
\frac{d}{d t}\left\|\omega_{h}\right\|^{2} \leq 0
$$

which becomes an equality if the central flux (1.9) is used.

The stability for the velocity field is now straightforward: we take $\varphi=\psi_{h}$ in (1.7) to obtain:

$$
\left\langle\nabla \psi_{h} \cdot \nabla \psi_{h}\right\rangle=-\left\langle\omega_{h} \psi_{h}\right\rangle \leq\left\|\psi_{h}\right\|\left\|\omega_{h}\right\| \leq C\left\|\nabla \psi_{h}\right\|\left\|\omega_{h}\right\|
$$

by the Poincare inequality, which implies

$$
\left\|\mathbf{u}_{h}\right\|=\left\|\nabla \psi_{h}\right\| \leq C\left\|\omega_{h}\right\| .
$$

Indeed, we can obtain a total energy conservation through the following arguments. Taking $v=\psi_{h}$ in (1.6), we obtain

$$
\left\langle\partial_{t} \omega_{h} \psi_{h}\right\rangle_{K^{\prime}}-\left\langle\omega_{h} \mathbf{u}_{h} \cdot \nabla \psi_{h}\right\rangle_{K}+\sum_{\rho \in \partial K}\left\langle\mathbf{u}_{h} \cdot \mathbf{n} \widehat{\omega_{h}} \psi_{h}\right\rangle_{e}=0
$$

Now the second term is zero since $\mathbf{u}_{h} \cdot \nabla \psi_{h}=0$. The third term vanishes after summing over all elements since $\psi_{h}$ is continuous. Finally, noticing that

$$
-\left\langle\partial_{t} \omega_{h} \psi_{h}\right\rangle=\frac{d}{d t} \frac{1}{2}\left\|\nabla \psi_{h}\right\|^{2}=\frac{d}{d t} \frac{1}{2}\left\|\mathbf{u}_{h}\right\|^{2},
$$

we obtain the conservation of energy

$$
\frac{d}{d t}\left\|\mathbf{u}_{h}\right\|=0
$$

even for a upwind flux. Thus there is no numerical dissipation for the energy.

We now turn to the error estimates. For these we would need to assume that the solution is regular. Conceptionally, since this is a finite element method, the exact solution of the PDE satisfies the scheme exactly. As usual, we define the two projection operators: $P$ is the standard $L^{2}$ projection into the space $V_{h}^{k}$; and $\Pi$ is the standard projection into $W_{0, h}^{k}$ :

$$
\langle\nabla(\psi-\Pi \psi) \cdot \nabla \varphi\rangle=0, \quad \forall \varphi \in W_{0 . h}^{-k} .
$$

Denote the error functions by

$$
\varepsilon=\omega-\omega_{h}, \quad \delta=\psi-\psi_{h}
$$


and their projections by

$$
\varepsilon_{h}=P \varepsilon=P \omega-\omega_{h}, \quad \delta_{h}=\Pi \delta=\Pi \psi-\psi_{h} .
$$

We first obtain a control of $\delta_{h}$ in terms of $\varepsilon$ :

$$
\langle\nabla \delta \cdot \nabla \varphi\rangle=-\langle\varepsilon \varphi\rangle, \quad \forall \varphi \in W_{0, h}^{k}
$$

from the scheme (1.7) and the fact that the exact solution also satisfies (1.7). Now, taking $\varphi=\delta_{h}$, we obtain

$$
\left\langle\nabla \delta_{h} \cdot \nabla \delta_{h}\right\rangle=\left\langle\nabla \delta \cdot \nabla \delta_{h}\right\rangle=-\left\langle\varepsilon \delta_{h}\right\rangle,
$$

which gives

$$
\left\|\nabla \delta_{h}\right\| \leq C\|\varepsilon\|
$$

This leads to a bound for the velocity field

$$
\begin{aligned}
\left\|\mathbf{u}-\mathbf{u}_{h}\right\|=\left\|\nabla\left(\psi-\psi_{h}\right)\right\| & \leq\|\nabla(\psi-\Pi \psi)\|+\left\|\nabla\left(\Pi \psi-\psi_{h}\right)\right\| \\
& \leq\|\nabla(\psi-\Pi \psi)\|+C\|\varepsilon\| .
\end{aligned}
$$

Since both the numerical solution and the exact solution satisfy (1.6),

$$
\left\langle\partial_{\ell} \varepsilon v\right\rangle_{K}-\left\langle\left(\omega \mathbf{u}-\omega_{h} \mathbf{u}_{h}\right) \cdot \nabla v\right\rangle_{K}+\sum_{\epsilon \in \partial K^{\prime}}\left\langle\left(\mathbf{u} \cdot \mathbf{n} \omega-\mathbf{u}_{h} \cdot \mathbf{n} \widehat{\omega_{h}}\right) v^{-}\right\rangle_{e}=0, \quad \forall v \in V_{h}^{k}
$$

Take $v=\varepsilon_{h}$. The second term becomes

$$
\left\langle\left(\omega \mathbf{u}-\omega_{h} \mathbf{u}_{h}\right) \cdot \nabla \varepsilon_{h}\right\rangle_{K}=\left\langle\omega\left(\mathbf{u}-\mathbf{u}_{h}\right) \cdot \nabla \varepsilon_{h}\right\rangle_{K}+\left\langle\varepsilon \mathbf{u}_{h} \cdot \nabla \varepsilon\right\rangle_{K}-\left\langle\varepsilon \mathbf{u}_{h} \cdot \nabla\left(\omega-P \omega^{\prime}\right)\right\rangle_{K} .
$$

Noticing that $\mathbf{u}-\mathbf{u}_{h}$ is exactly divergence free, we may perform integration by parts to the first term on the right side of (2.7) to obtain

$$
\left\langle\omega\left(\mathbf{u}-\mathbf{u}_{h}\right) \cdot \nabla \varepsilon_{h}\right\rangle_{K}=-\left\langle\varepsilon_{h}\left(\mathbf{u}-\mathbf{u}_{h}\right) \cdot \nabla \omega\right\rangle_{K}+\sum_{e \in \partial K^{\prime}}\left\langle\left(\mathbf{u}-\mathbf{u}_{h}\right) \cdot \mathbf{n} \omega \varepsilon_{h}^{-}\right\rangle_{\rho} .
$$

The second term on the right side of (2.7) is a complete derivative, hence can be integrated to give a pure boundary term

$$
\left\langle\varepsilon \mathbf{u}_{h} \cdot \nabla \varepsilon\right\rangle_{K}=\frac{1}{2} \sum_{\epsilon \in \partial K^{\circ}}\left\langle\mathbf{u}_{h} \cdot \mathbf{n}\left(\varepsilon^{-}\right)^{2}\right\rangle_{e}
$$

Plugging all these into (2.6) with $v=\varepsilon_{h}$, and collecting boundary terms, we obtain

$$
\left\langle\partial_{t} \varepsilon_{h} \varepsilon_{h}\right\rangle_{K}+\left\langle\varepsilon_{h}\left(\mathbf{u}-\mathbf{u}_{h}\right) \cdot \nabla \omega\right\rangle_{K}+\left\langle\varepsilon \mathbf{u}_{h} \cdot \nabla(\omega-P \omega)\right\rangle_{K}+\sum_{e \in \partial K} I_{e}=0
$$

where the boundary terms

$$
\begin{aligned}
I_{e} & =-\left\langle\left(\mathbf{u}-\mathbf{u}_{h}\right) \cdot \mathbf{n} \omega \varepsilon_{h}^{-}\right\rangle_{e}-\frac{1}{2}\left\langle\left(\mathbf{u}_{h} \cdot \mathbf{n}\left(\varepsilon^{-}\right)^{2}\right\rangle_{e}+\left\langle\left(\mathbf{u} \cdot \mathbf{n} \omega-\mathbf{u}_{h} \cdot \mathbf{n} \widehat{\omega_{h}}\right) \varepsilon_{h}^{-}\right\rangle_{e}\right. \\
& =\left\langle\mathbf{u}_{h} \cdot \mathbf{n}\left(\widehat{\varepsilon} \varepsilon_{h}^{-}-\frac{1}{2}\left(\varepsilon^{-}\right)^{2}\right)\right\rangle_{e} \\
& =\left\langle\mathbf{u}_{h} \cdot \mathbf{n}\left(\widehat{\varepsilon} \varepsilon^{-}-\frac{1}{2}\left(\varepsilon^{-}\right)^{2}\right)\right\rangle_{e}-\left\langle\mathbf{u}_{h} \cdot \mathbf{n} \widehat{\varepsilon}\left(\omega-(P \omega)^{-}\right)\right\rangle_{e} .
\end{aligned}
$$


Using the stability analysis in (2.2), we are left with

$$
\begin{aligned}
\frac{d}{d t} \frac{1}{2}\left\|\varepsilon_{h}\right\|^{2} \leq \sum_{K}\{ & -\left\langle\varepsilon_{h}\left(\mathbf{u}-\mathbf{u}_{h_{1}}\right) \cdot \nabla \omega\right\rangle_{K}-\left\langle\varepsilon \mathbf{u}_{h} \cdot \nabla(\omega-P \omega)\right\rangle_{K} \\
& \left.+\sum_{r \in i\rangle h}\left\langle\mathbf{u}_{h} \cdot \mathbf{n} \hat{\varepsilon}\left(\omega-(P \omega)^{-}\right)\right\rangle_{e}\right\}
\end{aligned}
$$

Assuming for the moment

$$
\left\|\mathbf{u}_{h}\right\|_{\infty} \leq C
$$

we can first estimate the boundary term

$$
\begin{aligned}
\sum_{K} \sum_{e \in \partial K}\left\langle\mathbf{u}_{h} \cdot \mathbf{n} \hat{\varepsilon}\left(\omega-(P \omega)^{-}\right)\right\rangle_{e} & \leq \sum_{K} \sum_{e \in \partial K} C\|[P \omega]\|_{e}\|\hat{\varepsilon}\|_{e} \\
& \leq\|\varepsilon\|^{2}+\frac{C}{h} \sum_{K} \sum_{e \in \partial K}\|[P \omega]\|_{e}^{2}
\end{aligned}
$$

Using the above inequality together with (2.5) and (2.8), we now obtain

$$
\left.\frac{d}{d t}\left\|\varepsilon_{h}\right\|^{2} \leq C\left(\left\|\varepsilon_{h}\right\|^{2}+\| \nabla(\psi-\Pi \psi\rangle\right)\left\|^{2}+\right\| \omega-P \omega\left\|_{H^{1}}^{2}+\frac{1}{h} \sum_{K} \sum_{e \in \partial K}\right\|[P \omega] \|_{e}^{2}\right) .
$$

Here we understand the norms as a summation of the same norm on each $K$. Using the standard interpolation theory [5], we obtain

$$
\frac{d}{d t}\left\|\varepsilon_{h}\right\|^{2} \leq C\left\|\varepsilon_{h}\right\|^{2}+C h^{2 k}
$$

which yields

$$
\left\|\varepsilon_{h}\right\| \leq C h^{k}
$$

Together with $(2.5)$, we have

$$
\left\|\mathbf{u}-\mathbf{u}_{h}\right\|+\left\|\omega-\omega_{h}\right\| \leq C h^{k} .
$$

Using an inverse inequality, we have

$$
\left\|\mathbf{u}-\mathbf{u}_{h}\right\|_{\infty} \leq C h^{k-1}
$$

this justifies the a priori assumption (2.9).

The estimate (2.9) is optimal in terms of the space $W_{0, h}^{k}$, which is important since the main cost for the scheme is in the Poisson solver in $W_{0 . h}^{k}$. The vorticity estimate in (2.9) is however suboptimal with respect to the space $V_{h}^{k}$. If we use $W_{0, h}^{k+1}$ instead for the streamfunction and the upwind flux (1.10), then a more detailed analysis will produce an order $O\left(h^{k+\frac{1}{2}}\right)$ for the error in $\omega$, see [21] and [12] for details. However, we do not recommend this choice in practice, as the increase of half order accuracy is obtained with the price of one degree higher polynomials in the most expensive part of the algorithm, namely the Poisson solver. In our numerical experiments in Sect. 5, we observe that close to $(k+1)$-th order of accuracy is generally achieved when $k$-th degree polynomials are used in both the discontinuous space for $\omega$ and the continuous space for $\psi$, both for uniform and for non-uniform meshes. 
3. The Scheme for the Navier-Stokes Equations. For the Navier-Stokes equations (1.3), there are two additional ingredients needing our attention:

1. The viscous terms cannot be directly implemented in the discontinuous space $V_{h}^{k}$. Instead, the stress tensor is first obtained locally using the same discontinuous Galerkin framework.

2. Vorticity boundary values are not known physically. We obtain vorticity boundary conditions locally from the streamfunction using the kinematic relation in (1.3).

We use the same finite element spaces $V_{h}^{k}$ and $W_{0, h}^{k}$ defined in (1.5) for the vorticity and streamfunction, respectively. Denote, by $V_{0, h}^{k}$, the subspace of $V_{h}^{k}$ with zero value at the boundary nodes. Let $W_{h}^{k}$ be the finite element spaces extended from $W_{0, h}^{k}$ with general non-zero values at the boundary nodes. The numerical method now becomes:

$$
\left\langle\partial_{t} \omega_{h} v\right\rangle_{K^{-}}-\left\langle\omega_{h} \mathbf{u}_{h} \cdot \nabla v\right\rangle_{K}+\sum_{e \in \partial K^{\prime}}\left\langle\mathbf{u}_{h} \cdot \mathbf{n} \widehat{\omega_{h}} v^{-}\right\rangle_{e}=-\left\langle\sigma_{h} \cdot \nabla v\right\rangle_{K}+\sum_{e \in \partial K^{\prime}}\left\langle\widetilde{\sigma_{h}} \cdot \mathbf{n} v^{-}\right\rangle_{\rho}, \quad \forall v \in \mathbf{I}_{0 . h}^{-k}
$$

Notice that the test function is now in $V_{0, h}^{k}$, (see [15]), and the stress tensor $\sigma_{h} \in\left(V_{h}^{k}\right)^{2}$ is obtained from the vorticity $\omega_{h}$ by the same discontinuous Galerkin framework:

$$
\operatorname{Re}\left\langle\sigma_{h} \mathbf{v}\right\rangle_{K}=-\left\langle\omega_{h} \nabla \cdot \mathbf{v}\right\rangle_{K}+\sum_{e \in \partial K^{K}}\left\langle\widetilde{\omega_{h}} \mathbf{v}^{-} \cdot \mathbf{n}\right\rangle_{e}, \quad \forall \mathbf{v} \in\left(V_{h}^{k}\right)^{2}
$$

The fluxes $\widetilde{\sigma_{h}}$ and $\widetilde{\omega_{h}}$ can be chosen as central averages

$$
\widetilde{\sigma_{h}}=\frac{1}{2}\left(\sigma_{h}^{-}+\sigma_{h}^{+}\right), \quad \widetilde{\omega_{h}}=\frac{1}{2}\left(\omega_{h}^{-}+\omega_{h}^{+}\right)
$$

or better still, as alternate one-sided fluxes, namely, at each edge $e$ with an arbitrarily fixed orientation, one of $\widetilde{\sigma_{h}}$ and $\widetilde{\omega_{h}}$ is taken as the left value and the other taken as the right value. It can be verified that, for $k=0$ and a rectangular triangulation, the central fluxes (3.3) produce a wide stencil central approximation to the second derivatives $\left(\omega_{i-2}, \omega_{i}\right.$ and $\omega_{i+2}$ are used for approximating $\omega_{x x}$ ), while the alternate one-sided fluxes produce a compact stencil central approximation $\left(\omega_{i-1}, \omega_{i}\right.$ and $\omega_{i+1}$ are used for approximating $\left.\omega_{x x}\right)$. Also, numerical and theoretical evidence shows that the alternate one-sided fluxes produce more accurate results [12]. In this paper we use only the alternate one-sided fluxes for the viscous terms.

We advocate the same steps as in [15] for a finite element method. In the time stepping, we first compute the vorticity at the interior nodes, and we will use these values to compute a stream function and then we use the stream function to determine the vorticity at the boundary nodes. This time-stepping is very efficient and we do not need any iteration between the boundary vorticity value and interior values, thus eliminating some traditional difficulties associated with the vorticity formulation. This time-stepping was first developed for finite-differences in $[13,14]$.

Since (3.1) is treated explicitly, The value of $\omega^{n+1}$ is computed via two steps. First, we compute $\left\langle\omega_{h}^{n+1} v\right\rangle_{K}$ for all $v \in V_{0, h}^{k}$ from the explicitly time stepping of (3.1). The value of $\omega_{h}^{n+1}$ at the interior element can now be directly computed from this term. However, the value of $\omega_{h}^{n+1}$ at the boundary element shall be determined after we computed the stream function as we explain below.

These values, $\left\langle\omega_{h}^{n+1} v\right\rangle_{K}$ for all $v \in V_{0, h}^{k}$, is sufficient to be used to compute the stream function from

$$
-\left\langle\nabla \psi_{h}^{n+1} \cdot \nabla \varphi\right\rangle=\left\langle\omega_{h}^{n+1} \varphi\right\rangle, \quad \forall \varphi \in W_{0, h}^{k},
$$

with the velocity field obtained from the stream function by

$$
\mathbf{u}_{h}^{n+1}=\nabla^{\perp} \psi_{h}^{n+1} .
$$


We now describe how to get the vorticity at the boundary cells. Since $\psi_{h}^{n+1}$ is known, we can compute

$$
-\left\langle\nabla \psi_{h}^{n+1} \cdot \nabla \varphi\right\rangle=\left\langle\omega_{h}^{n+1} \varphi\right\rangle
$$

for the test function $\varphi$ at the boundary nodes. We can then use it to compute the value of vorticity at the boundary elements.

For problems with periodic boundary conditions, the formulation above admits the following stability results:

$$
\frac{d}{d t}\left\|\omega_{h}\right\|^{2}+2\left\|\sigma_{h}\right\| \leq 0
$$

which in turn implies stability for the velocity field (2.3). The proof is similar to the Euler case, see [12] for the details. With the vorticity boundary condition mentioned above, we are unable to obtain a stability estimate. However, this type of vorticity boundary treatment for conventional finite difference and finite elements is stable, see [14] and [15].

4. Accuracy Check and Numerical Examples. We implement our method on triangulations based on rectangles. When a $P^{k}$ result is referred to it is obtained with $P^{k}$ elements for the vorticity $\omega$ and $Q^{k}$ dements for the streamfunction $\psi$. Strictly speaking $Q^{k}$ elements should also be used for the vorticity $\omega$ for the exact energy conservation (2.4) to hold, however to save cost we use $P^{k}$ elements for the vorticity $\omega$ instead. Energy stability (2.3) and enstrophy stability (2.2) still hold in this case. We have used both the upwind flux (1.10) and the (global) Lax-Friedrichs flux (1.11) for the calculations, however we will only show the results obtained with the Lax-Friedrichs flux to save space. The time discretization is by the third order positive Runge-Kutta methods in [26].

Example 1: This example is used to check the accuracy of our schemes, both for the Euler equations (1.1) and for the Navier-Stokes equations (1.3) with $R e=100$, for both the periodic and the Dirichlet boundary conditions, and with both a uniform mesh and a non-uniform mesh. The non-uniform mesh is obtained by alternating between $0.9 \Delta x$ and $1.1 \Delta x$ for the mesh sizes in the $x$ direction, similarly for the mesh sizes in the $y$ direction. The initial condition is taken as

$$
\omega(x, y, 0)=-2 \sin (x) \sin (y),
$$

which was used in [4]. The exact solution for this case is known:

$$
\omega(x, y, t)=-2 \sin (x) \sin (y) e^{-\frac{2 t}{R_{e}}} .
$$

We use the domain $[0,2 \pi] \times[0,2 \pi]$ for the periodic case and $[0, \pi] \times[0, \pi]$ for the Dirichlet case and compute the errors at $t=2$ for the periodic case and at $t=1$ for the Dirichlet case. We list in Table 4.1 (uniform mesh) and Table 4.2 (non-uniform mesh) the $L_{1}$ and $L_{\infty}$ errors, at $t=2$, measured at the center of the cells, for the periodic boundary conditions. Table 4.3 (uniform mesh) and Table 4.4 (non-uniform mesh) contain the results with the Dirichlet boundary conditions at $t=1$. We remark that, because of the difference in the sizes of the domains of the periodic and Dirichlet cases, the errors with the same number of cells are of different values, but the orders of accuracy are similar. We have also computed the errors of the relevant derivatives at the centers of the cells, which help in giving us truly $L_{\infty}$ errors throughout the domain. We will not show them to save space. 
TABLE 4.1

Accuracy test, uniform meshes, periodic boundary conditions.

\begin{tabular}{|c|c|c|c|c|c|c|c|c|}
\hline & \multicolumn{4}{|c|}{ Euler } & \multicolumn{4}{|c|}{ Navier-Stokes with $R e=100$} \\
\hline mesh & $L^{1}$ error & order & $L^{\infty}$ error & order & $L^{1}$ error & order & $L^{\infty}$ error & order \\
\hline \multicolumn{9}{|c|}{ P1 } \\
\hline $16^{2}$ & $7.77 \mathrm{E}-03$ & $\cdots$ & $1.80 \mathrm{E}-02$ & $\cdots$ & $7.65 \mathrm{E}-03$ & -- & $1.82 \mathrm{E}-02$ & $\ldots$ \\
\hline $32^{2}$ & $1.01 \mathrm{E}-03$ & 2.94 & $2.46 \mathrm{E}-03$ & 2.87 & $1.03 \mathrm{E}-03$ & 2.89 & $2.55 \mathrm{E}-03$ & 2.83 \\
\hline $64^{2}$ & $1.28 \mathrm{E}-04$ & 2.99 & $3.14 \mathrm{E}-04$ & 2.97 & $1.36 \mathrm{E}-04$ & 2.92 & $3.44 \mathrm{E}-04$ & 2.89 \\
\hline $128^{2}$ & $1.60 \mathrm{E}-05$ & 3.00 & $3.94 \mathrm{E}-05$ & 2.99 & $1.80 \mathrm{E}-05$ & 2.92 & $4.63 \mathrm{E}-05$ & 2.89 \\
\hline \multicolumn{9}{|c|}{$\mathrm{P} 2$} \\
\hline $16^{2}$ & $6.26 \mathrm{E}-04$ & - & $1.58 \mathrm{E}-03$ & $\ldots$ & $2.06 \mathrm{E}-(04$ & - & $5.85 \mathrm{E}-(04$ & - \\
\hline $32^{2}$ & $5.52 \mathrm{E}-0.5$ & 3.50 & $2.75 \mathrm{E}-(04$ & 2.52 & $1.37 \mathrm{E}-05$ & 3.90 & $3.24 \mathrm{E}-05$ & 4.17 \\
\hline $64^{2}$ & $4.82 \mathrm{E}-06$ & 3.52 & $3.81 \mathrm{E}-05$ & 2.85 & $2.40 \mathrm{E}-06$ & 2.51 & $4.10 \mathrm{E}-06$ & 2.98 \\
\hline $128^{2}$ & $4.04 \mathrm{E}-07$ & 3.58 & $4.96 \mathrm{E}-06$ & 2.94 & $4.05 \mathrm{E}-07$ & 2.57 & $6.44 \mathrm{E}-07$ & 2.67 \\
\hline \multicolumn{9}{|c|}{ P3 } \\
\hline $16^{2}$ & $9.74 \mathrm{E}-05$ & - & $2.31 \mathrm{E}-04$ & - & $9.68 \mathrm{E}-05$ & -- & $2.33 \mathrm{E}-04$ & - \\
\hline $32^{2}$ & $6.81 \mathrm{E}-06$ & 3.84 & $1.67 \mathrm{E}-05$ & 3.79 & $6.22 \mathrm{E}-06$ & 3.96 & $1.50 \mathrm{E}-05$ & 3.96 \\
\hline $64^{2}$ & $4.36 \mathrm{E}-07$ & 3.96 & $1.05 \mathrm{E}-06$ & 3.99 & $3.82 \mathrm{E}-07$ & 4.02 & $9.25 \mathrm{E}-07$ & 4.02 \\
\hline $128^{2}$ & $2.71 \mathrm{E}-08$ & 4.01 & $6.59 \mathrm{E}-08$ & 3.99 & $2.33 \mathrm{E}-(08$ & 4.04 & $5.70 \mathrm{E}-08$ & 4.02 \\
\hline
\end{tabular}

TABLE 4.2

Accuracy test, non-uniform meshes, periodic boundary conditions.

\begin{tabular}{|c|c|c|c|c|c|c|c|c|}
\hline & \multicolumn{4}{|c|}{ Euler } & \multicolumn{4}{|c|}{ Navier-Stokes with $R e=100$} \\
\hline mesh & $L^{1}$ error & order & $L^{\infty}$ error & order & $L^{1}$ error & order & $L^{\infty}$ error & order \\
\hline \multicolumn{9}{|c|}{ P1 } \\
\hline $16^{2}$ & $8.49 \mathrm{E}-03$ & -- & $2.85 \mathrm{E}-02$ & - & $7.77 \mathrm{E}-03$ & $\ldots$ & $2.80 \mathrm{E}-02$ & $-\ldots$ \\
\hline $32^{2}$ & $1.44 \mathrm{E}-03$ & 2.56 & $5.56 \mathrm{E}-03$ & 2.36 & $1.16 \mathrm{E}-03$ & 2.75 & $5.45 \mathrm{E}-03$ & 2.36 \\
\hline $64^{2}$ & $2.81 \mathrm{E}-04$ & 2.36 & $1.13 \mathrm{E}-03$ & 2.29 & $2.17 \mathrm{E}-04$ & 2.42 & $1.03 \mathrm{E}-03$ & 2.40 \\
\hline $128^{2}$ & $5.90 \mathrm{E}-05$ & 2.25 & $2.59 \mathrm{E}-04$ & 2.13 & $4.13 \mathrm{E}-05$ & 2.40 & $1.94 \mathrm{E}-04$ & 2.41 \\
\hline \multicolumn{9}{|c|}{$\mathrm{P} 2$} \\
\hline $16^{2}$ & $7.88 \mathrm{E}-04$ & - & $2.77 \mathrm{E}-03$ & $\cdots$ & $3.37 \mathrm{E}-04$ & - & $1.18 \mathrm{E}-03$ & - \\
\hline $32^{2}$ & $7.82 \mathrm{E}-05$ & 3.33 & $4.11 \mathrm{E}-04$ & 2.75 & $1.78 \mathrm{E}-05$ & 4.24 & $6.40 \mathrm{E}-05$ & 4.21 \\
\hline $64^{2}$ & $7.66 \mathrm{E}-06$ & 3.35 & $5.15 \mathrm{E}-05$ & 3.00 & $2.63 \mathrm{E}-06$ & 2.76 & $6.97 \mathrm{E}-06$ & 3.20 \\
\hline $128^{2}$ & $7.43 \mathrm{E}-07$ & 3.37 & $6.11 \mathrm{E}-06$ & 3.07 & $4.34 \mathrm{E}-07$ & 2.60 & $1.00 \mathrm{E}-06$ & 2.80 \\
\hline \multicolumn{9}{|c|}{ P3 } \\
\hline $16^{2}$ & $1.03 \mathrm{E}-04$ & - & $3.26 \mathrm{E}-04$ & - & $1.01 \mathrm{E}-04$ & - & $3.24 \mathrm{E}-04$ & - \\
\hline $32^{2}$ & $7.18 \mathrm{E}-06$ & 3.84 & $2.60 \mathrm{E}-05$ & 3.65 & $6.52 \mathrm{E}-06$ & 3.96 & $2.14 \mathrm{E}-05$ & 3.92 \\
\hline $64^{2}$ & $4.60 \mathrm{E}-07$ & 3.96 & $1.77 \mathrm{E}-06$ & 3.88 & $4.01 \mathrm{E}-07$ & 4.02 & $1.28 \mathrm{E}-06$ & 4.06 \\
\hline $128^{2}$ & $2.86 \mathrm{E}-08$ & 4.01 & $1.09 \mathrm{E}-07$ & 4.02 & $2.44 \mathrm{E}-08$ & 4.03 & $7.70 \mathrm{E}-08$ & 4.06 \\
\hline
\end{tabular}


TABLE 4.3

Accuracy test, uniform meshes, Dirichlet boundary conditions.

\begin{tabular}{|c|c|c|c|c|c|c|c|c|}
\hline & \multicolumn{4}{|c|}{ Euler } & \multicolumn{4}{|c|}{ Navier-Stokes with $R e=100$} \\
\hline mesh & $L^{1}$ error & order & $L^{\times}$error & order & $L^{1}$ error & order & $L^{\infty}$ error & order \\
\hline \multicolumn{9}{|c|}{ P1 } \\
\hline $16^{2}$ & $5.92 \mathrm{E}-04$ & -- & $1.23 \mathrm{E}-03$ & - & $5.75 \mathrm{E}-04$ & - & $1.32 \mathrm{E}-03$ & - \\
\hline $32^{2}$ & $8.19 \mathrm{E}-05$ & 2.85 & $1.92 \mathrm{E}-04$ & 2.68 & $7.52 \mathrm{E}-05$ & 2.94 & $1.78 \mathrm{E}-04$ & 2.89 \\
\hline $64^{2}$ & $1.06 \mathrm{E}-05$ & 2.94 & $5.35 \mathrm{E}-05$ & 1.84 & $9.63 \mathrm{E}-06$ & 2.96 & 0 & 2.25 \\
\hline $128^{2}$ & $1.35 \mathrm{E}-(06$ & 8 & 1 & 1.92 & $1.25 \mathrm{E}-06$ & $x$ & & 2.22 \\
\hline \multicolumn{9}{|c|}{ P2 } \\
\hline $16^{2}$ & $.76 \mathrm{E}-05$ & - & $2.57 \mathrm{E}-04$ & - & $1.51 \mathrm{E}-05$ & 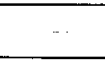 & $4.05 \mathrm{E}-05$ & - \\
\hline $32^{2}$ & $4.28 \mathrm{E}-06$ & 3.47 & $3.57 \mathrm{E}-05$ & 2.85 & $2.49 \mathrm{E}-06$ & 2.60 & $6.09 \mathrm{E}-06$ & 2.73 \\
\hline $64^{2}$ & $3.74 \mathrm{E}-07$ & 3.52 & $4.65 \mathrm{E}-06$ & 2.94 & $4.11 \mathrm{E}-07$ & 2.60 & $9.42 \mathrm{E}-07$ & 2.69 \\
\hline $128^{2}$ & $3.17 \mathrm{E}-08$ & 3.56 & $5.92 \mathrm{E}-07$ & 2.97 & $6.16 \mathrm{E}-08$ & 2.74 & $1.34 \mathrm{E}-07$ & 2.81 \\
\hline \multicolumn{9}{|c|}{$\mathrm{P3}$} \\
\hline $16^{2}$ & $6.80 \mathrm{E}-06$ & $\cdots$ & $1.58 \mathrm{E}-05$ & - & $6.34 \mathrm{E}-06$ & - & $1.53 \mathrm{E}-05$ & - \\
\hline $32^{2}$ & $4.22 \mathrm{E}-07$ & 4.01 & $1.06 \mathrm{E}-06$ & 3.90 & $3.90 \mathrm{E}-07$ & 4.02 & $9.45 \mathrm{E}-07$ & 4.02 \\
\hline $64^{2}$ & $2.66 \mathrm{E}-08$ & 3.99 & $6.90 \mathrm{E}-08$ & 3.94 & $2.38 \mathrm{E}-08$ & 4.04 & $5.81 \mathrm{E}-08$ & 4.02 \\
\hline $128^{2}$ & $1.66 \mathrm{E}-09$ & 4.00 & $4.25 \mathrm{E}-09$ & 4.02 & $1.46 \mathrm{E}-09$ & 4.03 & $3.59 \mathrm{E}-09$ & 4.02 \\
\hline
\end{tabular}

TABLE 4.4

Accuracy test, non-uniform meshes, Dirichlet boundary conditions.

\begin{tabular}{|c|c|c|c|c|c|c|c|c|}
\hline & \multicolumn{4}{|c|}{ Euler } & \multicolumn{4}{|c|}{ Navier-Stokes with $R e=100$} \\
\hline mesh & $L^{1}$ error & order & $L^{\infty}$ error & order & $L^{1}$ error & order & $L^{\infty}$ error & order \\
\hline \multicolumn{9}{|c|}{$\mathrm{P} 1$} \\
\hline $16^{2}$ & $1.12 \mathrm{E}-03$ & - & $4.35 \mathrm{E}-03$ & - & $9.93 \mathrm{E}-04$ & - & $4.25 \mathrm{E}-03$ & - \\
\hline $32^{2}$ & $2.44 \mathrm{E}-04$ & 2.20 & $9.79 \mathrm{E}-04$ & 2.15 & $1.95 \mathrm{E}-04$ & 2.35 & $8.74 \mathrm{E}-04$ & 2.28 \\
\hline $64^{2}$ & $5.61 \mathrm{E}-05$ & 2.12 & $2.39 \mathrm{E}-04$ & 2.04 & $3.90 \mathrm{E}-05$ & 2.33 & $1.75 \mathrm{E}-04$ & 2.32 \\
\hline $128^{2}$ & $1.36 \mathrm{E}-05$ & 2.04 & $6.29 \mathrm{E}-05$ & 1.92 & $7.76 \mathrm{E}-06$ & 2.33 & $3.54 \mathrm{E}-05$ & 2.31 \\
\hline \multicolumn{9}{|c|}{$\mathrm{P} 2$} \\
\hline $16^{2}$ & $7.54 \mathrm{E}-05$ & - & $3.31 \mathrm{E}-04$ & - & $1.98 \mathrm{E}-05$ & $\cdots$ & $6.63 \mathrm{E}-05$ & - \\
\hline $32^{2}$ & $8.15 \mathrm{E}-06$ & 3.21 & $4.33 \mathrm{E}-05$ & 2.93 & $2.61 \mathrm{E}-06$ & 2.93 & 7.03E-06 & 3.24 \\
\hline $64^{2}$ & $8.46 \mathrm{E}-07$ & 3.27 & $5.35 \mathrm{E}-06$ & 3.02 & $4.35 \mathrm{E}-07$ & 2.59 & $1.02 \mathrm{E}-06$ & 2.79 \\
\hline $128^{2}$ & $8.31 \mathrm{E}-08$ & 3.35 & $6.56 \mathrm{E}-07$ & 3.03 & $6.52 \mathrm{E}-08$ & 2.74 & $1.49 \mathrm{E}-07$ & 2.78 \\
\hline \multicolumn{9}{|c|}{$\mathrm{P3}$} \\
\hline $16^{2}$ & $7.17 \mathrm{E}-06$ & - & $2.49 \mathrm{E}-05$ & - & $6.65 \mathrm{E}-06$ & - & $2.18 \mathrm{E}-05$ & - \\
\hline $32^{2}$ & $4.46 \mathrm{E}-07$ & 4.01 & $1.66 \mathrm{E}-06$ & 3.91 & $4.09 \mathrm{E}-07$ & 4.02 & $1.31 \mathrm{E}-06$ & 4.06 \\
\hline $64^{2}$ & $2.80 \mathrm{E}-08$ & 3.99 & $1.04 \mathrm{E}-07$ & 3.99 & $2.50 \mathrm{E}-08$ & 4.03 & $7.86 \mathrm{E}-08$ & 4.06 \\
\hline $128^{2}$ & $1.75 \mathrm{E}-09$ & 4.00 & $6.89 \mathrm{E}-09$ & 3.92 & $1.53 \mathrm{E}-09$ & 4.02 & $4.77 \mathrm{E}-09$ & 4.04 \\
\hline
\end{tabular}


We can clearly see from these tables that close to $(k+1)$-th order of accuracy is generally achieved when $k$-th degree polynomials are used in both the discontinuous space for $\omega$ and for the Poisson solver, both for the uniform and for the non-uniform meshes.

Example 2: The double shear layer problem taken from [1]. We solve the Euler equation (1.1) in the domain $[0,2 \pi] \times[0,2 \pi]$ with a periodic boundary condition and an initial condition:

$$
\omega(x, y, 0)= \begin{cases}\delta \cos (x)-\frac{1}{\rho} \operatorname{sech}^{2}((y-\pi / 2) / \rho) & y \leq \pi \\ \delta \cos (x)+\frac{1}{\rho} \operatorname{sech}^{2}((3 \pi / 2-y) / \rho) & y>\pi\end{cases}
$$

where we take $\rho=\pi / 15$ and $\delta=0.05$.

The solution quickly develops into roll-ups with smaller and smaller scales, so on any fixed grid the full resolution is lost eventually. We use fixed uniform meshes of $64 \times 64$ and $128 \times 128$ rectangles and perform the calculation up to $t=8$. We plot the time history of total energy (square of the $L^{2}$ norm of velocity $\mathbf{u}$ ) and total enstrophy (square of the $L^{2}$ norm of vorticity $\omega$ ) in Fig. 4.1, as well as contours of the vorticity $\omega$ at $t=6$ in Fig. 4.2 and at $t=8$ in Fig. 4.3 to show the resolution. We can see from Fig. 4.1 that the numerical dissipation decreases roughly in the order of $P^{1} 64^{2}, P^{1} 128^{2}, P^{2} 64^{2}, P^{3} 64^{2}, P^{2} 128^{2}$, and $P^{3} 128^{2}$. The higher order methods have better resolutions and in general the resolution is quite good judging from the contours. We remark that when the numerical viscosity becomes too small with higher order methods, since the schemes are linear, numerical oscillations are unavoidable when resolution to sharp fronts is lost, leading to instability. This is common for all linear schemes. However, the discontinuous Galerkin method we use here is able to get stable solutions for much sharper fronts with the same mesh than central type finite difference or finite element methods. More extensive numerical resolution study for this example can be found in [23]. For a comparison with nonlinear ENO schemes, we refer to [16].
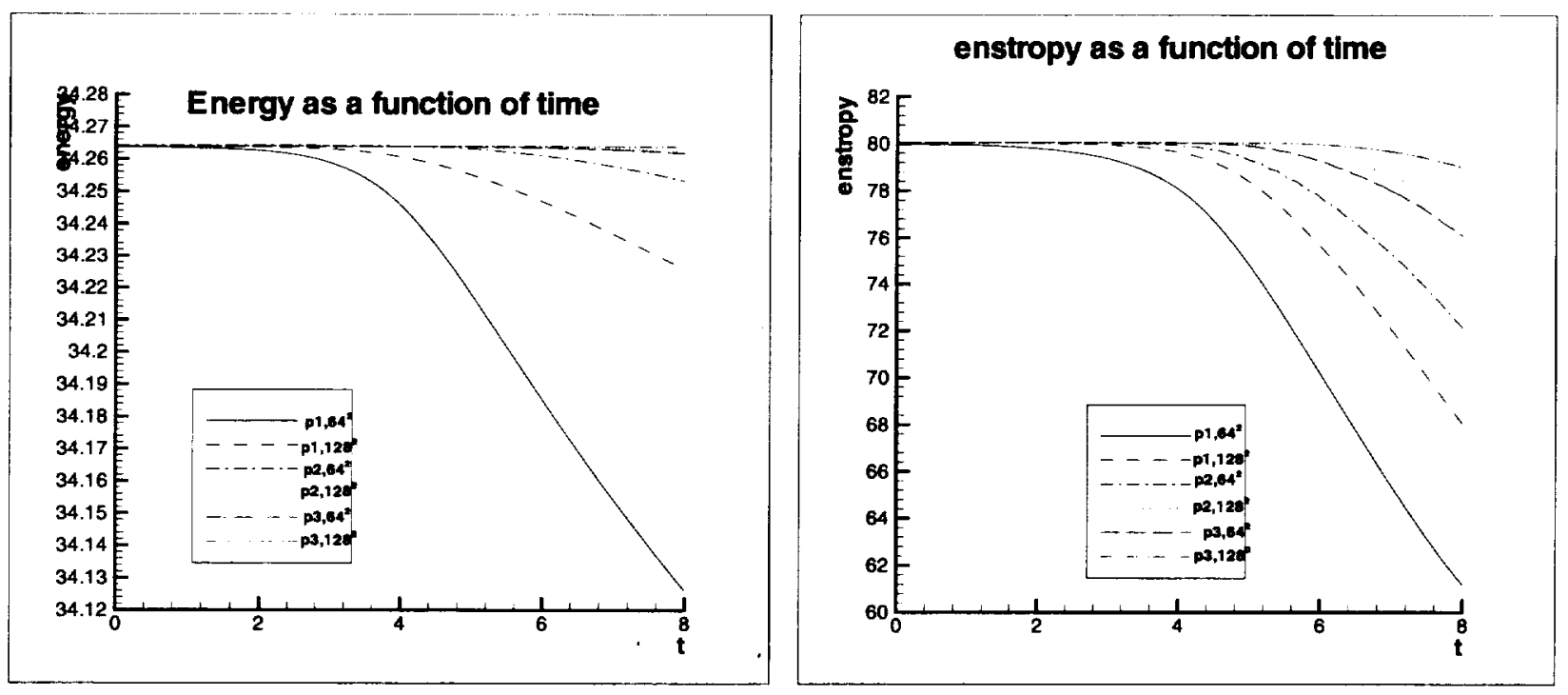

FIG. 4.1. The time history of energy (square of the $L_{2}$ norm of the velocity $\mathbf{u}$ ) and total enstrophy (square of the $L^{2}$ norm of vorticity $\omega$ ). $P^{1}$ with $64^{2}$ mesh in solid line, $P^{1}$ with $128^{2}$ mesh in dashed line, $P^{2}$ with $64^{2}$ mesh in dash-dot line, $P^{2}$ with $128^{2}$ mesh in dotted line, $P^{3}$ with $64^{2}$ mesh in long dashed line, and $P^{3}$ with $128^{2}$ mesh in dash-dot-dot line.

Example 3: The vortex patch problem. We solve the Euler equation $(1.1)$ in $[0,2 \pi] \times[0,2 \pi]$ with the 
following initial condition:

$$
\omega(x, y, 0)= \begin{cases}-1, & \frac{\pi}{2} \leq x \leq \frac{3 \pi}{2}, \quad \frac{\pi}{4} \leq y \leq \frac{3 \pi}{4} \\ 1, & \frac{\pi}{2} \leq x \leq \frac{3 \pi}{2}, \quad \frac{5 \pi}{4} \leq y \leq \frac{7 \pi}{4} \\ 0, & \text { otherwise }\end{cases}
$$

and periodic boundary conditions. The contour plots of vorticity $\omega$, with 30 equally spaced contour lines between $\omega=-1.1$ and $\omega=1.1$, are given in Fig. 4.4 for $t=5$ and in Fig. 4.5 for $t=10$. We can see that the scheme gives stable results for all runs, and higher order schemes give better resolutions for vorticity.

5. Concluding Remarks. We have developed a high order discontinuous Galerkin method for the two dimensional incompressible Euler and Navier-Stokes equations in the vorticity streamfunction formulation, coupled with a standard continuous finite element solution of the Poisson equation for the streamfunction. A natural matching between the two finite element spaces allows us to obtain total energy conservation and total enstrophy stability. Numerical examples are shown to demonstrate the accuracy and resolution of the methods.

Acknowledgments. This work was initialized when both authors were visiting the Mittag-Leffler Institute in Sweden. We thank the faculty and staff there for their warm hospitality.

\section{REFERENCES}

[1] J. Beil, P. Colella and H. Glaz, A second order projection method for the incompressible NavierStokes equations, J. Comput. Phys., 85 (1989), pp. 257-283.

[2] R. Biswas, K.D. Dfvine AND J. FlaherTY, Parallel, adaptive finite element methods for conservation laws, Appl. Numer. Math., 14 (1994), pp. 255-283.

[3] A. ChORIN, A numerical method for solving incompressible viscous flow problems, J. Comput. Phys., 2 (1967), pp. 12-26.

[4] A. Chorin, Numerical solution of the Navier-Stokes equations, Math. Comp., 22 (1968), pp. 745-762.

[5] P. Ciarlet, The Finite Element Method for Elliptic Problems, North Holland, 1975.

[6] B. Cockburn, An introduction to the discontinuous Galerkin method for convection-dominated problems, in Advanced Numerical Approximation of Nonlinear Hyperbolic Equations, B. Bockburn, C. Johnson, C.-W. Shu and E. Tadmor (Editor: A. Quarteroni), Lecture Notes in Mathematics, volume 1697, Springer, 1998.

[7] B. Cockburn and C.-W. Shu, The Runge-Kutta local projection $P^{1}$-discontinuous Galerkin method for scalar conservation laws, $M^{2} A N, 25$ (1991), pp. 337-361.

[8] B. Cockburn and C.-W. Shu, TVB Runge-Kutta local projection discontinuous Galerkin finite element method for scalar conservation laws II: General framework, Math. Comp., 52 (1989), pp. 411-435.

[9] B. Cockburn, S.-Y. Lin And C.-W. Shu, TVB Runge-Kutta local projection discontinuous Galerkin finite element method for conservation laws III: One dimensional systems, J. Comput. Phys., 84 (1989), pp. 90-113.

[10] B. Cockburn, S. Hou, And C.-W. Shu, TVB Runge-Kutta local projection discontinuous Galerkin finite element method for conservation laws IV: The multidimensional case, Math. Comp., 54 (1990), pp. $545-581$. 
[11] B. Cockburn and C.-W. Shu, TVB Runge-Kutta local projection discontinuous Galerkin finite element method for scalar conservation laws V: Multidimensional systems, .J. Comput. Phys., 141 (1998), pp. 199224.

[12] B. CockBunn AND C.-W. SHu, The local discontinuous Galerkin method for time-dependent convection diffusion systems, SIA.M .J. Numer. Anal., 35 (1998), pp. 24402463.

[13] W. E AND J.-G. Lu, Vorticity boundary condition and related issues for finite difference schemes, J. Comput. Phys., 124 (1996), pp. 368382.

[14] W. E AND J.-G. LIU, Essentially compact schemes for unsteady viscous incompressible flows, J. Comput. Phys., 126 (1996), pp. 122-138.

[15] W. E And J.-G. Lı, An efficient finite element method for incompressible flows in vorticity-stream function formulation, preprint. Submitted to Math. Comp.

[16] W. E And C.-W. SHu, A numerical resolution study of high order essentially non-oscillatory schemes applied to incompressible flou, J. Comput. Phys., 110 (1994), p1). 3946.

[17] V. Girault and P.A. Raviat, Finite Element Methods for Navier-Stokes Equations, Theory and Algorithms, Springer-Verlag, Berlin, 1986.

[18] P.M. Gresho, Some interesting issues in incompressible fluid dynamics, both in the continuum and in numerical simulation, Advances in Applied Mechanics, 28 (1992), pp. 45140.

[19] M. Gunzburcier, Finite element methods for viscous incompressible flows, Academic Press, Boston, 1989.

[20] G. JIANG AND C.-W. SHU, On cell entropy inequality for discontinuous Galerkin methods, Math. Comp., 62 (1994), pp. 531-538.

[21] C. .Johnson And J. PItKäranta, An analysis of the discontinuous Galerkin method for a scalar. hyperbolic equation, Math. Comp., 46 (1986), pp. $1-26$.

[22] D. LeVY and E. TADMOR, Non-oscillatory central schemes for the incompressible 2-D Euler equations, Math. Research Letters, 4 (1997), pp. 1-20.

[23] J.-G. LIU AND C.-W. SHU, A numerical example on the performance of high order discontinuous Galerkin method for 2D incompressible flows, to appear in the Proceedings of the International Symposium on Discontinuous Galerkin Methods, Newport, Rhode Island, May 24-26, 1999. B. Cockburn, G. Karniadakis and C.-W. Shu, editors, Springer-Verlag.

[24] O.A. Pironneav, Finite Element Methods for Fluids, John Wiley and Sons, 1989.

[25] L. QUaRTAPElle, Numerical solution of the incompressible Navier-Stokes equations, Birkhäuser, Berlin, 1993.

[26] C.-W. SHU AND S. OsHER, Efficient implementation of essentially non-oscillatory shock-capturing schemes, J. Comput. Phys., 77 (1988), pp. 439-471.

[27] R. Temam, Navier-Stokes Equations, Theory and Numerical Analysis, North-Holland, New York, 1979. 

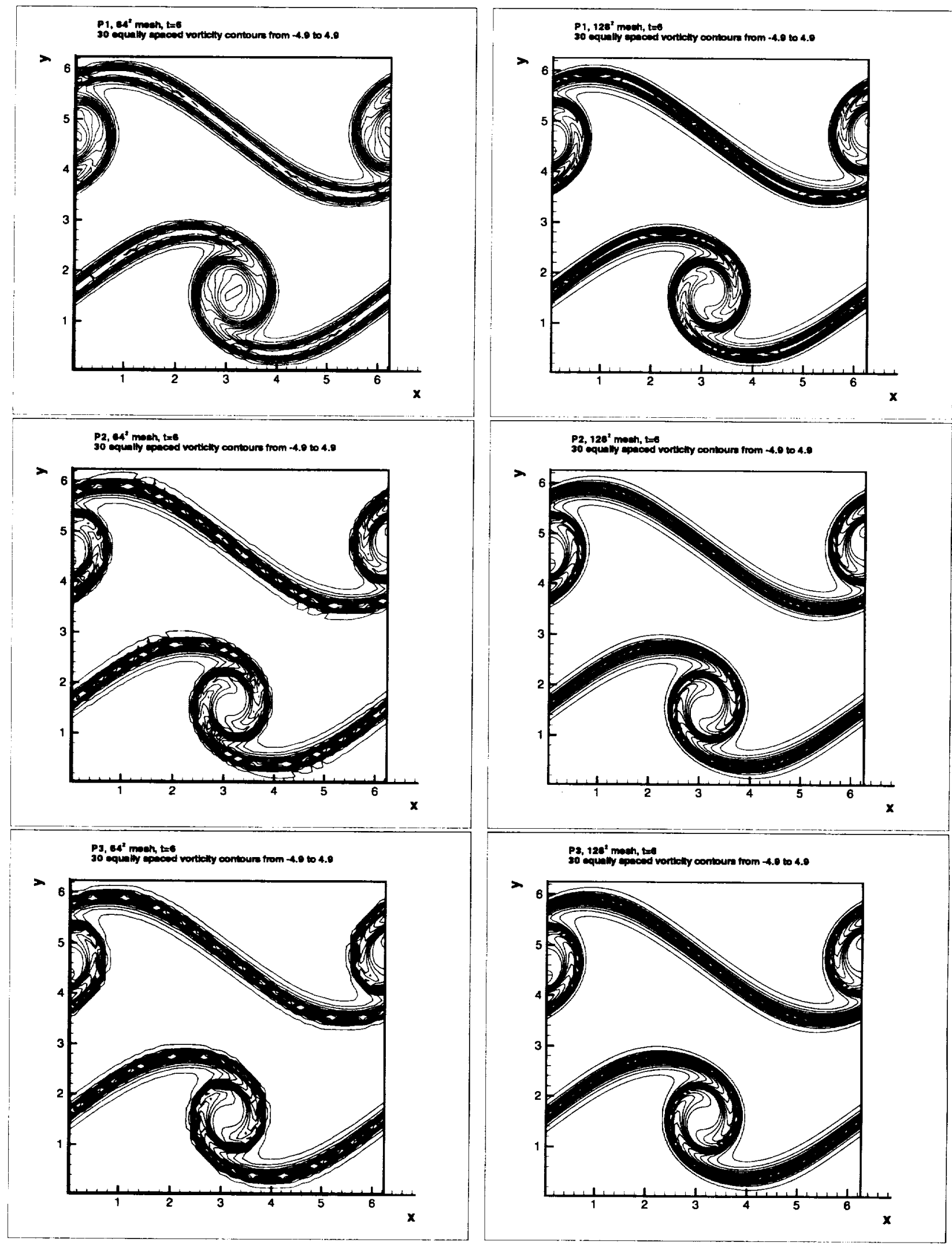

FiG. 4.2. Contour of vorticity $\omega$ at $t=6.30$ equally spaced contour lines between $\omega=-4.9$ and $\omega=4.9$. Left: results with $64^{2}$ mesh; Right: results with $128^{2}$ mesh. Top: $P^{1} ;$ middle: $P^{2}$, bottom: $P^{3}$. 


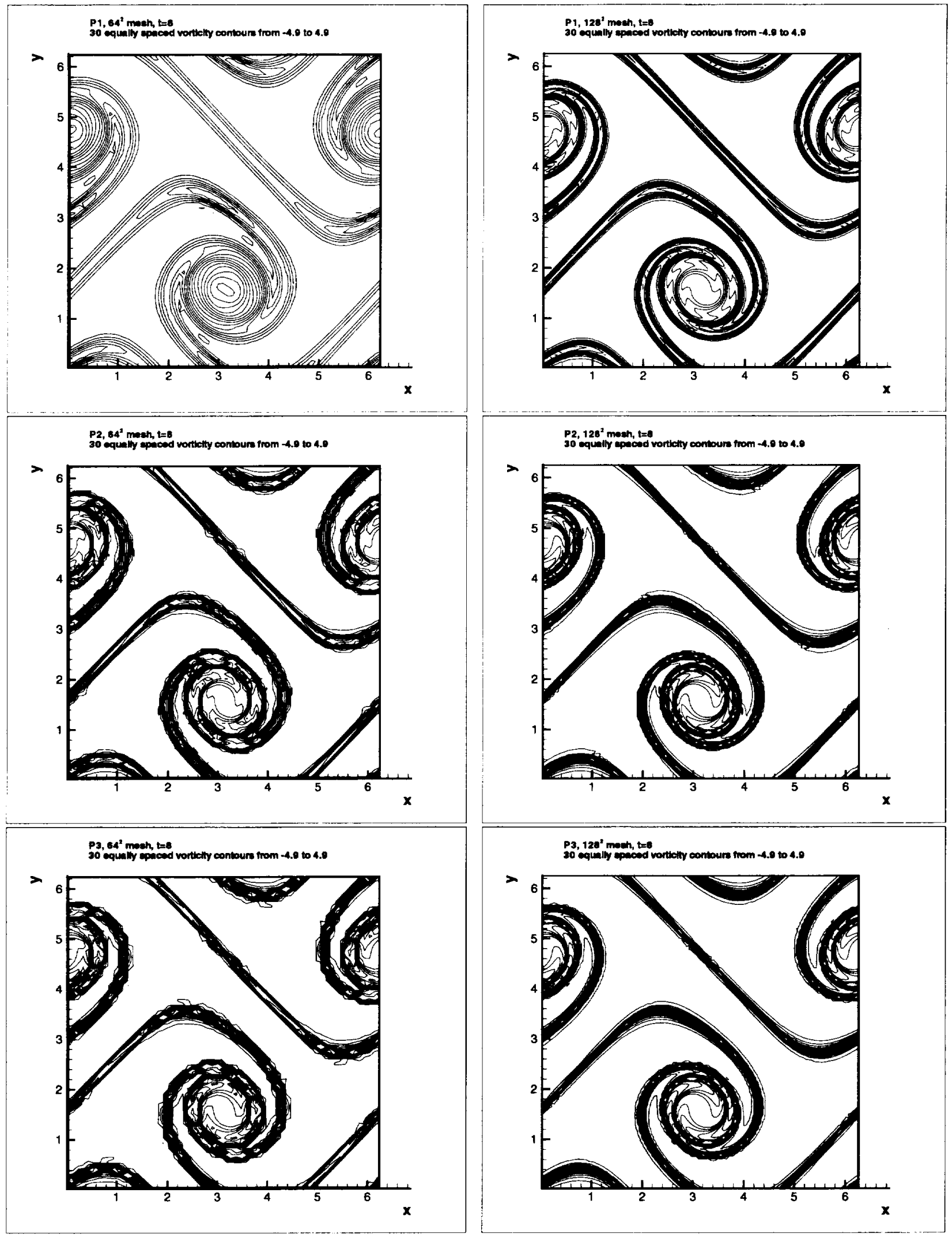

FiG. 4.3. Contour of vorticity $\omega$ at $t=8$. 30 equally spaced contour lines between $\omega=-4.9$ and $\omega=4.9$. Left: results with $64^{2}$ mesh; Right: results with $128^{2}$ mesh. Top: $P^{1}$; middle: $P^{2}$, bottom: $P^{3}$. 

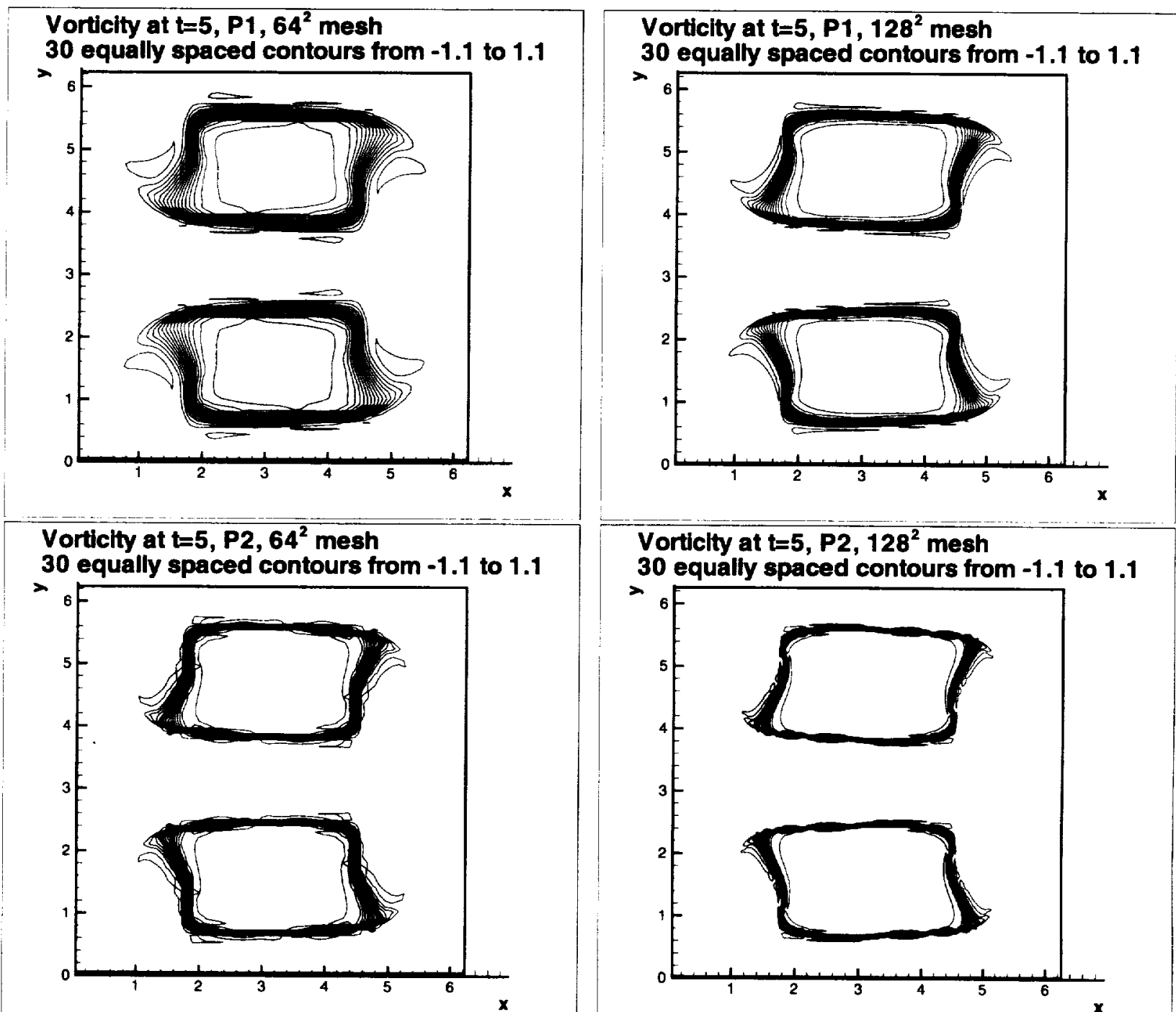

Vorticlty at $t=5, P 2,128^{2}$ mesh
30 equally spaced contours from -1.1 to 1.1

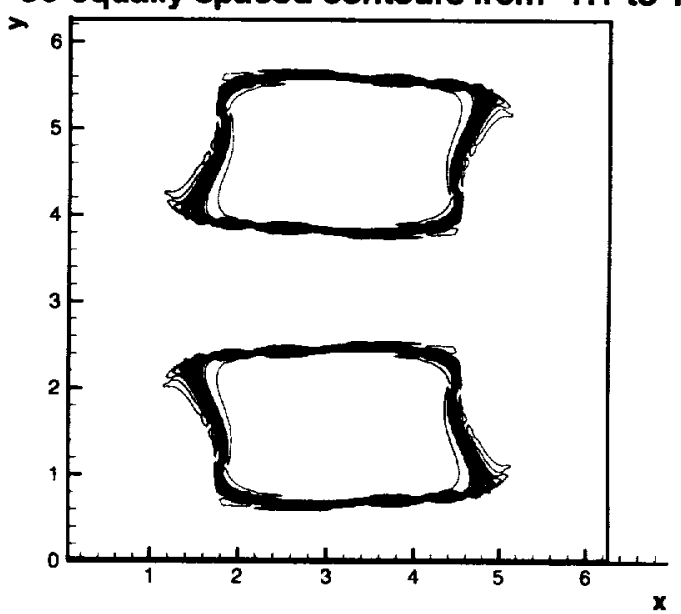

Vorticity at $t=5, P 3,64^{2}$ mesh
30 equally spaced contours from -1.1 to 1.1

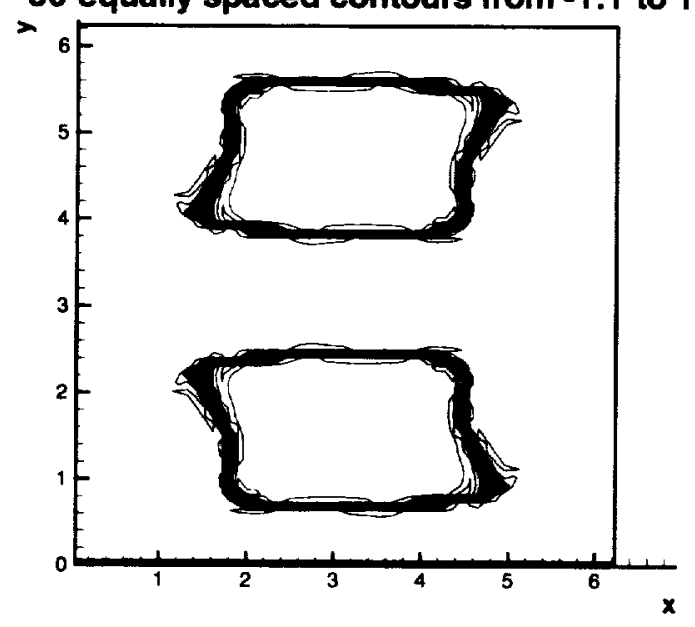

Vorticlty at $t=5, P 3,128^{2}$ mesh

30 equally spaced contours from -1.1 to 1.1

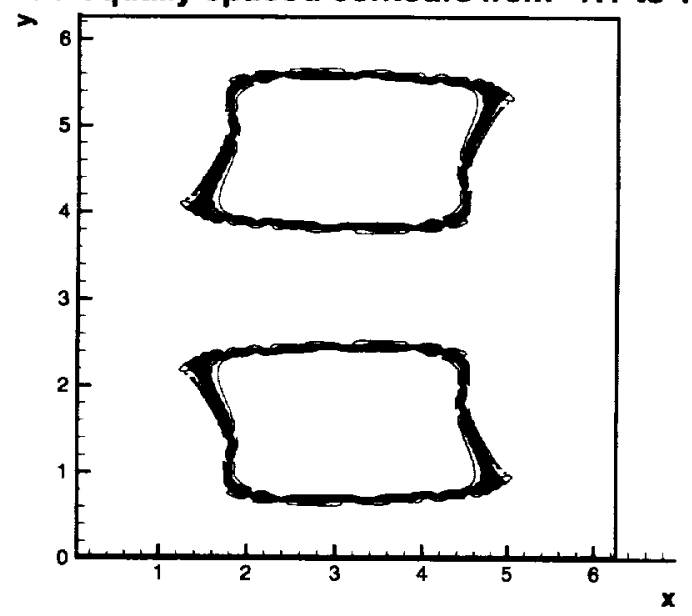

FIG. 4.4. Contour of vorticity $\omega$ at $t=5.30$ equally spaced contour lines between $\omega=-1.1$ and $\omega=1.1$. Left: results with $64^{2}$ mesh; Right: results with $128^{2}$ mesh. Top: $P^{1}$; middle: $P^{2}$, bottom: $P^{3}$. 


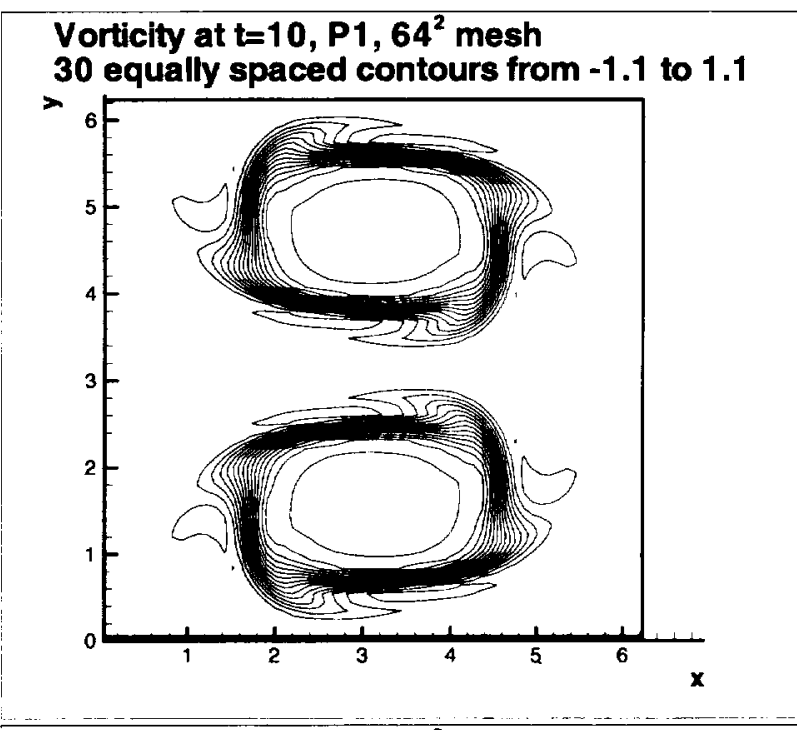

Vorticity at $t=10, P 2,64^{2}$ mesh

30 equally spaced contours from -1.1 to 1.1

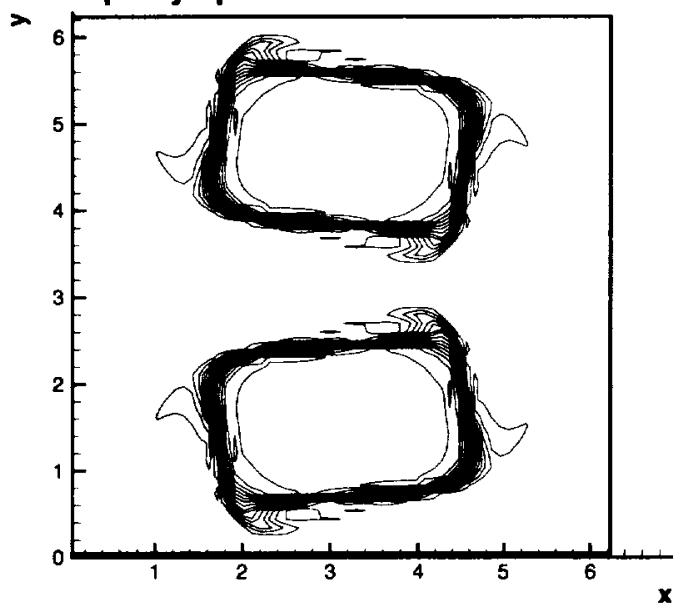

Vorticity at $\mathrm{t}=10, \mathrm{P3}, 64^{2}$ mesh

30 equally spaced contours from -1.1 to 1.1

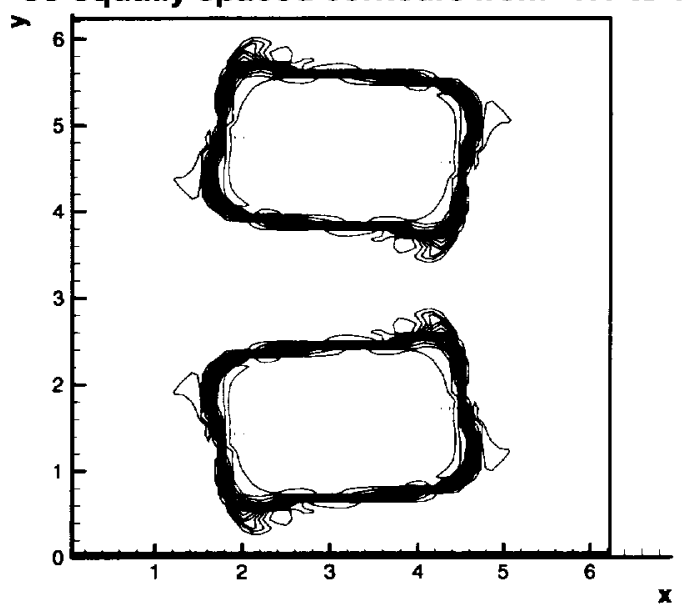

Vorticity at $t=10, P 1,128^{2}$ mesh

30 equally spaced contours from -1.1 to 1.1

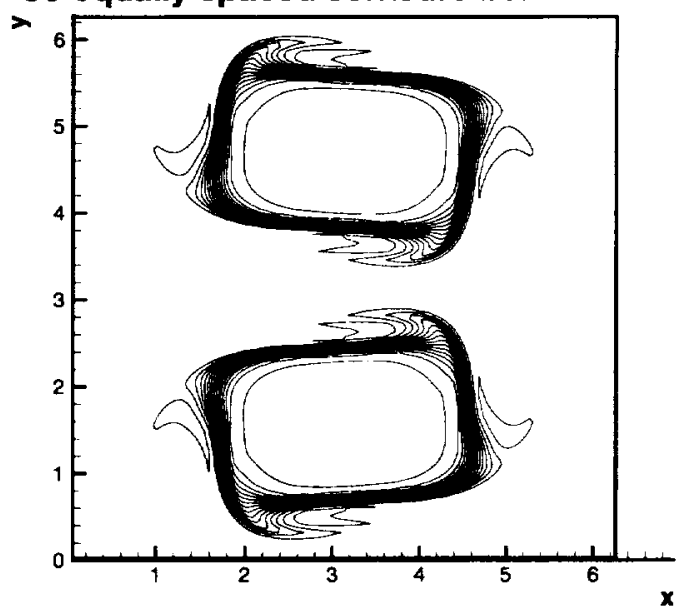

Vorticity at $t=10, P 2,128^{2}$ mesh 30 equally spaced contours from -1.1 to 1.1

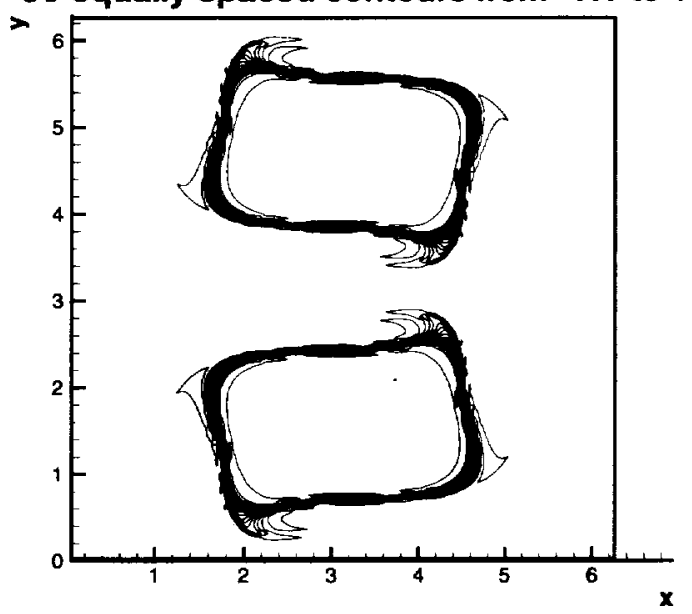

Vorticity at $t=10, P 3,128^{2}$ mesh 30 equally spaced contours from -1.1 to 1.1

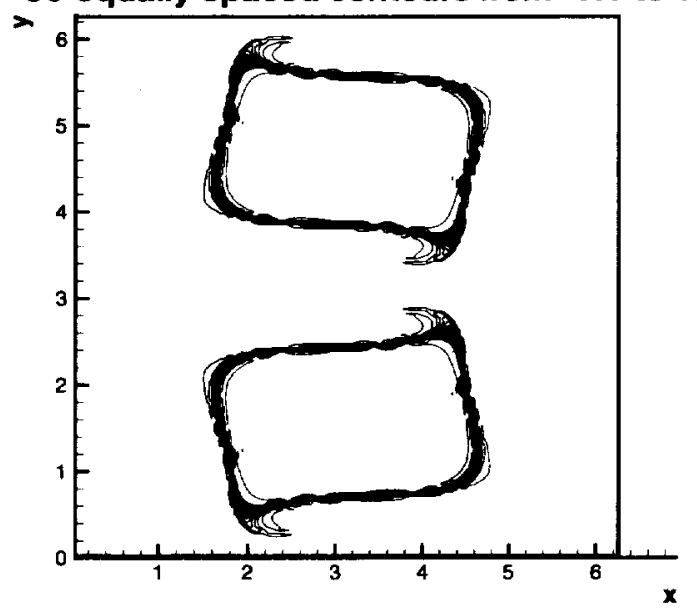

FIG. 4.5. Contour of vorticity $\omega$ at $t=10$. 30 equally spaced contour lines between $\omega=-1.1$ and $\omega=1.1$. Left: results with $64^{2}$ mesh; Right: results with $128^{2}$ mesh. Top: $P^{1}$; middle: $P^{2}$, bottom: $P^{3}$. 
Public reporting burden for this collection of information is estimated to average 1 hour per response, including the time for reviewing instructions, searching existing data sources, gathering and maintaining the data needed. and completing and reviewing the collection of information. Send comments regarding this burden estimate or any other aspect of this

\begin{tabular}{|l|l|l|}
\hline 1. AGENCY USE ONLY(Leave blank) & $\begin{array}{c}\text { 2. REPORT DATE } \\
\text { July } 1999\end{array}$ & $\begin{array}{l}\text { 3. REPORT TYPE AND DATES COVERED } \\
\text { Contractor Report }\end{array}$
\end{tabular}

\section{TITLE AND SUBTITLE}

5. FUNDING NUMBERS

A high order discontinuous Galerkin method for $2 \mathrm{D}$ incompressible

Hows

C. NAS1-97046

WU 505-90-52-01

\section{AUTHOR(S)}

Jian-Gino Lin

Chi- Wang Shu

7. PERforming ORgANization NAME(S) AND ADDRESS(ES)

Institute for Computer Applications in Science and Engineering

Mail Stop 132C, NASA Langley Research Center

Hampton, VA 23681-2199

8. PERFORMING ORGANIZATION

REPORT NUMBER

IC ASE Report No. $99-27$

9. SPONSORING/MONITORING AGENCY NAME(S) AND ADDRESS(ES)

National Aeronautics and Space Administration

Langley Research Centor

Hampton, VA 23681-2199

10. SPONSORING/MONITORING AGENCY REPORT NUMBER

NASA/CR-1999-209361

ICASE Report No. 99-27

11. SUPPLEMENTARY NOTES

Langley Technical Monitor: Demnis M. Bushnell

Final Report

Submitted to Journal of Computational Physics.

12a. DISTRIBUTION/AVAILABILITY STATEMENT

Unclassified Unlimited

Subject Category 64

Distribution: Nonstandard

Availability: NASA-CASI (301) 621-0390

13. ABSTRACT (Maximum 200 words)

In this paper we introduce a high order discontinuous Galerkin method for two dimensional incompressible tlow in vorticity streamfunction formulation. The momentum equation is treated explicitly, utilizing the efficiency of the discontinuous Galerkin method. The streamfunction is obtained by a standard Poisson solver using continuous finite elements. There is a natural matching between these two finite element spaces, since the normal component of the velocity field is continuous across element boundaries. This allows for a correct upwinding gluing in the discontinuous Galerkin framework, while still maintaining total energy conservation with no numerical dissipation and total enstrophy stability. The method is suitable for inviscid or high Reynolds number flows. Optimal error estimates are proven and verified by numerical experiments.

14. SUBJECT TERMS

incompressible flow, discontinuous Galerkin, high order accuracy

12b. DISTRIBUTION CODE

17. SECURITY CLASSIFICATION
OF REPORT
Unclassified

NSN 7540-01-280-5500

\section{SECURITY CLASSIFICATION OF THIS PAGE} Unclassified
19. SECURITY CLASSIFICATION OF ABSTRACT

\begin{tabular}{l} 
15. NUMBER OF PAGES \\
22 \\
\hline 16. PRICE CODE \\
A03 \\
\hline $\begin{array}{l}\text { 20. LIMITATION } \\
\text { OF ABSTRACT }\end{array}$ \\
Standard Form 2987(Rev. 2-89) \\
Prescribed by ANSI Std. Z39-18
\end{tabular}

